\title{
A Novel Spectral Zoom Method based on Zolotarev Polynomials
}

\author{
Jan Kubák and Pavel Sovka \\ Department of Circuit Theory \\ Faculty of Electrical Engineering (FEE) \\ Czech Technical University (CTU) in Prague \\ Prague, Czech republic \\ jankubak@gmail.com,sovka@fel.cvut.cz
}

\begin{abstract}
This paper discusses the usage of symmetrical Zolotarev polynomials (ZPS)s in spectral analysis. Evaluation of Discrete Zolotarev transform (DZT) coefficients is briefly discussed. However, the DZT coefficients evaluation is problematic. An alternative novel method embedding ZPSs is proposed. The novel method, so-called DZT zoom, improves the spectrum's time resolution for non-stationary signals compared to narrowband spectrogram. Results are comparable to the approximated DZT (ADZT). The DZT zoom distinguishing property is the focus on a particular frequency band of the spectrum.
\end{abstract}

Keywords-discrete Zolotarev transform, symmetrical Zolotarev polynomials, non-stationary spectral transform, approximated discrete Zolotarev transform

\section{INTRODUCTION}

Zolotarev polynomials (ZP)s have been studied for decades [1]-[5] for several reasons. One of the reasons is a possibility to design optimal digital FIR filters [6], [7]. Elliptic IIR filters are associated with both Zolotarev and Cauer [8]. Usage of ZPs in spectral analysis began with discrete Zolotarev transform (DZT) [9], which employs symmetrical ZP (ZPS) [10]. The DZT is a spectral transform intended for nonstationary signal analysis. The DZT coefficients have not yet been evaluated directly in the time domain. An alternative method employing approximated ZPs, so-called approximated DZT (ADZT), was developed. Properties of the ADZT are discussed and compared with other non-stationary signal analysis methods in [11]-[13].

The ADZT algorithm performs basis optimization in the spectral domain. However, the direct DZT spectrum evaluation in the time domain is still an important element missing in order to understand the DZT properties better. It is much more feasible to link features of the resulting spectrum to the basis properties in the time domain. Firstly, the parameters of the ZPSs are directly observable in the polynomial waveform. Secondly, ZPSs have only a single parameter, the modulus of elliptical functions, which is related to the time selectivity feature of the polynomials. On the other hand, the approximated ZPSs have two parameters: the non-stationary bandwidth and non-stationary index.

The DZT is defined in Sec. II-A. The direct evaluation of DZT coefficients is briefly explained in Sec. II-B. An alternative novel method based on the
ZPS, of which coefficients are evaluated in the time domain, is proposed in Sec. III. The novel method is compared with short-time discrete Fourier transform (STDFT) and short-time ADZT (STADZT) [11] in Sec. IV.

\section{Discrete Zolotarev Transform}

\section{A. Definition}

The DZT is described briefly for the purpose of explaining its basic principle. Radim Špetík's dissertation thesis [14] contains a detailed definition. The DZT basis is composed of ZPSs. The Zolotarev cosine (Zcos) and Zolotarev sine (Zsin) form real and imaginary parts of the basis, respectively [10]. The Zolotarev series [9] expansion can be defined analogically to trigonometric series expansion as:

$$
\begin{aligned}
& \mathrm{Z} \exp \left(\ell, k^{\prime}, i 2 \pi t\right)= \\
& \quad \mathrm{Z} \cos \left(\ell, k^{\prime}, 2 \pi t\right)+i \mathrm{Z} \sin \left(\ell, k^{\prime}, 2 \pi t\right)
\end{aligned}
$$

for $0 \leq t \leq 1$, where $\ell$ is degree of $\mathrm{ZP}$, and $0 \leq k^{\prime}<1$ is modulus of elliptical functions. For an illustration, an example of the real and imaginary parts of the basis is given in Fig. 1. The modulus of elliptical functions $k^{\prime}$ defines width of center section of the function definition interval, where the function does not have equiripple property. The central section forms central lobe of which width, height, and area are functions of $k^{\prime}$. Hence the modulus of elliptical functions $k^{\prime}$ controls the amount of time-selectivity of the basis. Note that the Zsin and Zcos are mutually almost orthogonal for all degrees $\ell$ and moduli of elliptical functions $k^{\prime}$ [14]:

$$
\left\langle\mathrm{Z} \cos \left(\ell, k^{\prime}, 2 \pi t\right), \mathrm{Z} \sin \left(\ell, k^{\prime}, 2 \pi t\right)\right\rangle \approx 0 \quad \forall k^{\prime}, \forall \ell .
$$

Since the energy of both the real and imaginary parts of the basis increase significantly for larger degrees for $k^{\prime}>0$ the parts are normalized separately. Normalization coefficients $\gamma$ are chosen such that following equation is satisfied for both parts:

$$
\begin{aligned}
\gamma_{R, \ell}^{2}\left\langle\mathrm{Z} \cos \left(\ell, k^{\prime}, 2 \pi t\right), \mathrm{Z} \cos \left(\ell, k^{\prime}, 2 \pi t\right)\right\rangle & =\frac{1}{2} \\
\gamma_{I, \ell}^{2}\left\langle\mathrm{Z} \sin \left(\ell, k^{\prime}, 2 \pi t\right), \mathrm{Z} \sin \left(\ell, k^{\prime}, 2 \pi t\right)\right\rangle & =\frac{1}{2}
\end{aligned}
$$




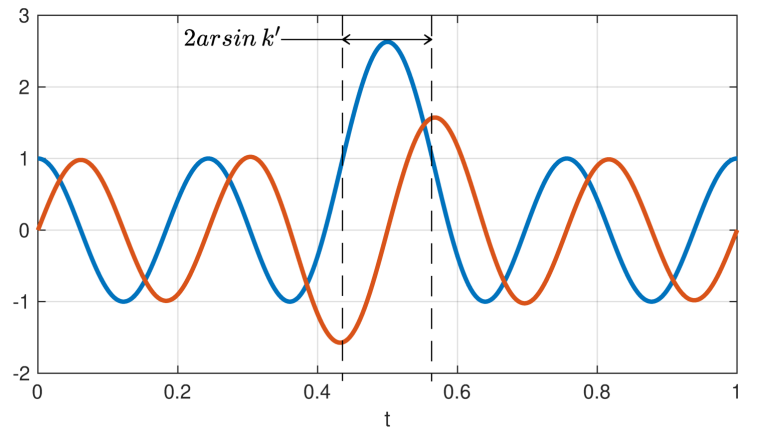

Fig. 1. An example of Zolotarev cosine $Z \cos (2 \pi t)$ (blue line) and sine $Z \sin (2 \pi t)$ (red line) for degree $\ell=4$ and modulus of elliptical functions $k^{\prime}=0.2$.

where $\gamma_{R}$ and $\gamma_{I}$ are normalization coefficients of the real and imaginary parts, respectively. The normalized Zolotarev exponential denotes as:

$$
\begin{aligned}
& \mathrm{Z} \exp ^{\prime}\left(\ell, k^{\prime}, i 2 \pi t\right)= \\
& \underbrace{\gamma_{R, \ell}^{2} \mathrm{Z} \cos }_{\mathrm{Z} \cos ^{\prime}}\left(\ell, k^{\prime}, \omega t\right)+i \underbrace{\gamma_{I, \ell}^{2} \mathrm{Z} \sin }_{\mathrm{Z} \sin ^{\prime}}\left(\ell, k^{\prime}, \omega t\right) .
\end{aligned}
$$

The normalized DZT basis is defined using discretized Zexp' in analogy to the DFT basis as:

$$
\begin{array}{r}
W_{N^{\prime}}^{\prime \ell, n}\left(k^{\prime}\right)=\frac{1}{\sqrt{N}} \operatorname{Zexp}^{\prime}\left(\ell, k^{\prime}, \frac{i 2 \pi n}{N}\right) \\
\ell, n=0 \ldots N-1 .
\end{array}
$$

The parameters $k^{\prime}$ is omitted in the $W$ parameters for simplicity. The value of $k^{\prime}$ is fixed for all $n$ but it can differ for different $\ell$. Finally, set of DZT coefficients, DZT spectrum, is given by the scalar product of the basis (5) and analyzed signal $s[n]$ as:

$$
Z^{\prime}[\ell]=\left\langle W_{N}^{\prime \ell, n}, s[n]\right\rangle \quad \ell, n=0 \ldots N-1,
$$

assuming $N$-point discrete signal $s[n], n=0 \ldots N-$ 1 .

\section{B. Coefficient Evaluation}

The direct evaluation of the DZT spectrum coefficients has not yet been achieved. There are two main reasons why. Firstly, generation of symmetric Zolotarev polynomials is non-trivial [15]. Secondly, energy of the Zexp (1) rises enormously with increasing degree $\ell$ for $k^{\prime}>0$. This is the reason for introduction of the normalization. The normalization (4) makes the basis (5) normal; however, the result of the scalar product (6) carries little signal information. The reason is that the normalization suppresses the equiripple, trigonometrical, part of the basis. The trigonometrical part exhibits the DFT behavior which is expected of the transform; this behavior is suppressed significantly, by the same amount as the energy of Zexp grows.

\section{An Alternative Novel Method}

\section{A. Principle}

The proposed novel method is based on the following reasoning.

- Firstly, let's define an assumption that the method shall exhibit DFT spectrum's global (long-term) properties. Such that a stationary signal result is similar to the DFT spectrum of the analyzed signal.

- Secondly, let the method reflect both the global and local spectral properties of the analyzed signal. The global signal energy properties are related to the signal long-term integral properties, interval within the signal can be considered stationary. The local signal energy properties are related to the signal envelope [12].

- Thirdly, the amount of the local analyzed signal property is given by the scalar product of analyzed signal and a selective basis function closely related to the ZPSs. This amount of local selectivity is called local selectivity index $K$.

- Fourthly, the local properties of the analyzed signal are imposed on the global properties. Thus, assuming the global analyzed signal spectral properties are represented in the DFT spectrum, the final result is the DFT spectrum multiplied by the local selectivity index $K$.

Now, let's illustrate the principle on an example. Assume an analyzed triangular signal with a different offset from the center of the analyzed window. The signal with energy centroid in the center is depicted in Figure 2a, with right of the center in Figure 2c, and with left of the center in Figure 2e. An illustration of the selective basis waveform is depicted as a dashed line in the figure. Note that for all analyzed signal shifts the resulting DFT spectrum is equal in its absolute value. Figure 2b, 2d, and $2 \mathrm{f}$ depict DFT spectrums for respective analyzed signal shifts; the blue lines denote the DFT spectrum amplitudes.

Consider that the DFT spectrum amplitude values are multiplied by the scalar product of the analyzed signal and the selective basis, the local selectivity index $K$. In the first case, where the energy centroid of the analyzed signal is at the center, the resulting spectrum is equal to the DFT spectrum. In the second case, where the energy centroid of the analyzed signal is right of the center, the resulting spectrum is reduced in amplitude by $1 / K$, see the red lines in the Figure $2 \mathrm{~d}$. Similarly, in the third case, see the red lines in Figure 2f. The local selectivity index $K$ is equal for both cases since the selective basis is even symmetric around the center. Results in the second and third case reflect that the local analyzed signal spectral properties are not aligned with the selective basis. Now assume that the resulting spectrum is composed similarly to the STDFT, using the windowing process. The output will exhibit additional resolution in time compared to the STDFT. The result is actually similar to the one of the STADZT.

\section{B. Selective Basis}

The selective basis of the proposed method is composed of, so-called, non-stationary part of the Zolotarev exponential Zexp (4). Note that there are more Zsin definitions. This work uses the definition according to [10]. The Zexp can be decomposed into non- 


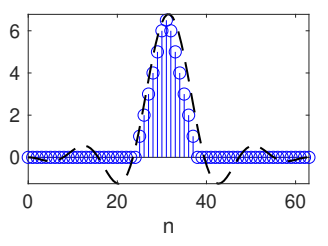

(a) Signal w1; energy at the window center is depicted as samples; selective basis waveform is depicted by the dashed line.

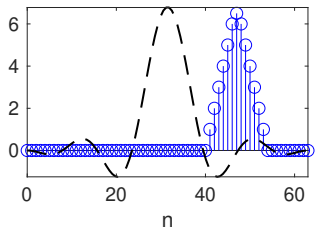

(c) Signal w2; shifted to the right of center is depicted as samples; selective basis waveform is depicted by the dashed line.

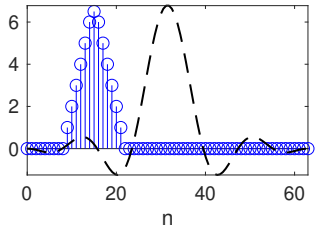

(e) Signal w3; shifted to the left of center is depicted as samples; selective basis waveform is depicted by the dashed line.

Fig. 2. An illustration of proposed ad hoc non-stationary DFT based method principle; an example triangular signal with different signal shifts is depicted in (a), (c), and (e). The absolute value of the DFT spectrum for each signal shift is depicted in (b), (d), and (f), respectively, by the blue samples; the resulting spectrum with imposed local spectral signal properties is depicted by the red samples.

stationary $(\mathcal{N})$ and stationary $(\mathcal{S})$ parts, see [9]. The non-stationary part $\mathcal{N}$ is decomposed as:

$$
\begin{aligned}
& \mathcal{N}\left\{\operatorname{Zexp}\left(\ell, k^{\prime}, i 2 \pi t\right)\right\}=\mathcal{N}\left(\ell, k^{\prime}\right)= \\
& \operatorname{Zexp}\left(\ell, k^{\prime}, i 2 \pi t\right)-\left.\mathrm{Z} \exp \left(\ell, k^{\prime}, i 2 \pi t\right)\right|_{k^{\prime}=0}= \\
& \operatorname{Zexp}\left(\ell, k^{\prime}, i 2 \pi t\right)-\underbrace{\exp (\ell, i 2 \pi t)}_{\mathcal{S}(\ell)} .
\end{aligned}
$$

The decomposition of the Zcos to the stationary and non-stationary parts is depicted in Fig. 3. Thus, the selective basis denoted as $\Delta$ is composed of the Zexp non-stationary part $\mathcal{N}(7)$ as:

$$
\Delta\left(\ell, k^{\prime}\right)=\mathcal{N}\left\{\operatorname{Zexp}\left(\ell, k^{\prime}, i 2 \pi t\right)\right\} .
$$

\section{Definition}

As outlined previously, the method weights the DFT spectrum of analyzed signal by the local selectivity index $K$. The $K$ is a scalar product of the selective basis $\Delta\left(m, k^{\prime}\right)(8)$ and and analyzed signal, in principle. However, the fact that the energy of symmetrical ZPs has large dynamic range (DR) has to be addressed. The high DR is mitigated by the introduced Zexp

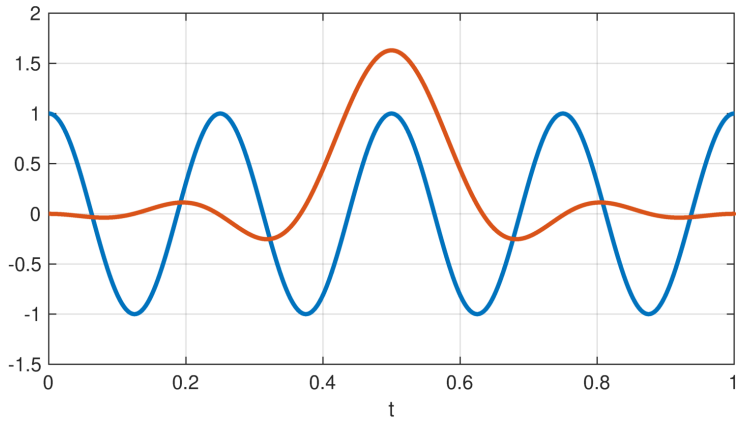

Fig. 3. Decomposition of Zolotarev cosine $Z \cos (2 \pi t)$ into stationary part $\mathcal{S}(\ell)$ (blue line) and non-stationary part $\mathcal{N}(\ell)$ (red line) for degree $\ell=4$ and modulus of elliptical functions $k^{\prime}=0.2$. The Zcos has the same parameters as in Fig. 1.

normalization (4). Additionally, the analyzed signal window energy has to be normalized as well. Both normalizations lead to computation of the local selectivity index $K$ as a correlation coefficient between the analyzed signal window and the selective basis $\Delta$ as:

$$
K\left(\ell, k^{\prime}\right)=\frac{\left\langle\Delta\left(\ell, k^{\prime}\right), s\right\rangle}{\mathrm{E}\left\{\Delta\left(\ell, k^{\prime}\right)\right\} \mathrm{E}\{s\}},
$$

where $\Delta\left(\ell, k^{\prime}\right)$ is the selective basis (8), $s$ is discrete analyzed signal of length $N$, and operator $\mathrm{E}\{$.$\} is$ signal energy operator. The result is then equal to the multiplication of the analyzed signal window DFT spectrum by the $K\left(\ell, k^{\prime}\right)$ as:

$$
\begin{array}{r}
\left|S_{Z}^{\prime \prime}(\ell)\right|=|\operatorname{DFT}\{s\}(\ell)| K\left(\ell, k^{\prime}\right) \\
\ell \subset\{0, \ldots, N-1\} .
\end{array}
$$

The spectrum is computed separately for the real and imaginary parts. The DFT spectrum leakage reduction can be employed by commonly used window functions without a limitation.

The proposed method does not perform any optimization of the selective basis; no optimal modulus of elliptical function $k^{\prime}$ is being found. Instead, the $k^{\prime}$ is a parameter of the method. The parameter $k^{\prime}$ is related to center frequency of zoomed-in frequency bandwidth, where the method provides improved time-resolution compared with the STDFT. The lower the value of $k^{\prime}$ the higher is the value of the center frequency. This feature is similar to, so-called, "zoom" technique [16], [17], where a desired frequency band is selected by a filter bank and consequentially zoomed in. Therefore, the proposed technique has been named "DZT zoom".

\section{RESULTS}

An example signal is used to compare the short-time DZT zoom spectrum with the STDFT and STADZT ones. The signal is comprised of two sine waves mixture, Gaussian pulse, and Dirac pulse, see Fig. 4. The wideband spectrogram, Fig. 5a, of the signal localizes the Gaussian and Dirac pulses well in time; however, the frequency resolution is relatively poor. The two sine waves mixture and the Dirac pulse are barely noticeable due to the DR of the spectrum. On the other hand, the narrowband spectrogram, Fig. 5b, has lower DR allowing to recognize the sine waves mixture in 


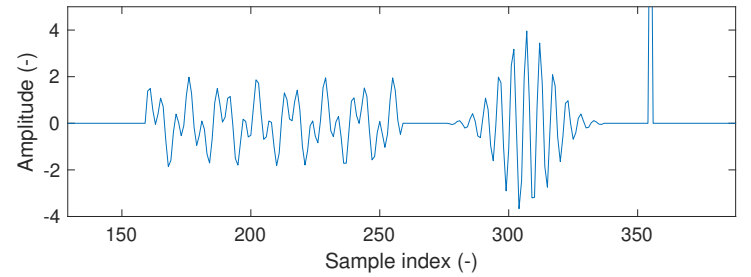

Fig. 4. Example analyzed signal composed of a mixture of two sine waves, Gaussian pulse, and Dirac pulse. The sine waves mixture has parameters of $f_{1}=75 \mathrm{~Hz}$ and $f_{2}=190 \mathrm{~Hz}$, same amplitude, and zero phase. The Gaussian pulse center frequency is $190 \mathrm{~Hz}$ and bandwidth is $0.2 \mathrm{~Hz}$. Sampling frequency of the signal is $1 \mathrm{kHz}$.

the spectrum. It has a higher frequency resolution, but particular parts of the signal are blurred together.

A non-stationary transform, such as STADZT, can be used to improve the spectrum resolutions for nonstationary signals. The STADZT spectrum of the signal, in Fig. 6, improves the time resolution while the same frequency resolution as of the narrowband spectrogram's is kept. Note that the ADZT is designed to work with rectangular signal weighting window. The STADZT removes certain parts of the spectrum performing hard decisions based on phase. As a consequence the spectrum becomes rather intermittent.

The DZT zoom short-time spectrum is depicted in Fig. 7a; the center focus frequency is set at $\approx 190 \mathrm{~Hz}$ by the modulus of elliptical functions $k^{\prime} \approx 0.04$. The center frequency is noticeable in local selectivity index $|K|$ gram, Fig. 7b, as the maximum of the Dirac pulse spectrum. The DZT zoom short-time spectrum of the Gaussian pulse is smooth compared to the intermittent STADZT spectrum. The intermittent spectrum of the Gaussian pulse is specific to the STADZT. On the other hand, the spectrum of the sine waves mixture is intermittent for both methods. The intermittent spectrum of harmonic, stationary, signals is a property of DZT based transforms. This property is related to the beat frequency present in the signal envelope formed by a mixture of harmonic signals. The selectivity index $K$ is correlated to the envelope beat frequency.

Sine wave of the lower frequency is barely noticeable in the DZT zoom short-time spectrum focused at $190 \mathrm{~Hz}$, Fig. 7a. Changing the center frequency at $\approx 80 \mathrm{~Hz}, k^{\prime}=0.07$, allows to recognize it in the spectrum, Fig. 8a. The center frequency is again noticeable in local selectivity index $|K|$ gram, Fig. 8b, as the maximum of the Dirac pulse spectrum.

The spectrum of the Dirac pulse is well localized in time by both methods.

\section{CONCLUSiON}

This article briefly describes the DZT and the difficulty of evaluating its coefficients. A novel method, so-called DZT zoom, based on the DZT is proposed. The DZT zoom uses the non-stationary part of the Zolotarev exponential as its basis. The novel method exhibits similar behavior to the ADZT. The spectrum time resolution is improved, the stationary signal's spectrum is intermittent. The intermittent spectrum of stationary harmonic signals is a property of DZT

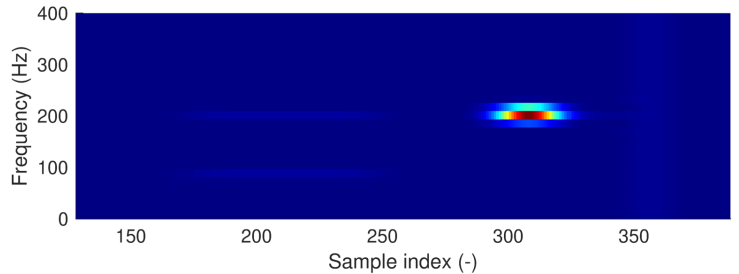

(a) Wideband spectrogram; window length of 64 samples.

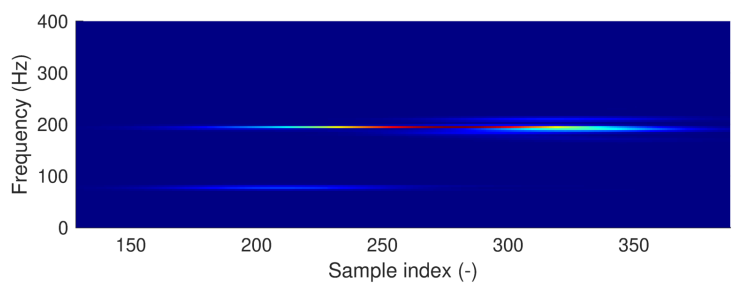

(b) Narrowband spectrogram; window length of 256 samples.

Fig. 5. STDFT spectrum modul of the signal Fig. 4 for various window lengths; Hamming window is used and window step is 1 sample for both spectrograms.

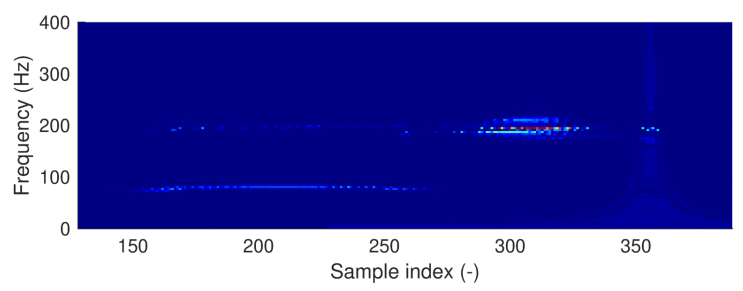

Fig. 6. STADZT spectrum modul of the analyzed signal Fig. 4; window length of 256 samples and window step of 1 sample; no weighting window is used.

based spectral methods. The important property of the novel transform is its focus on a specific frequency band. The band center frequency is set by a specific modulus of elliptical functions, which is a parameter of symmetrical Zolotarev polynomials. 


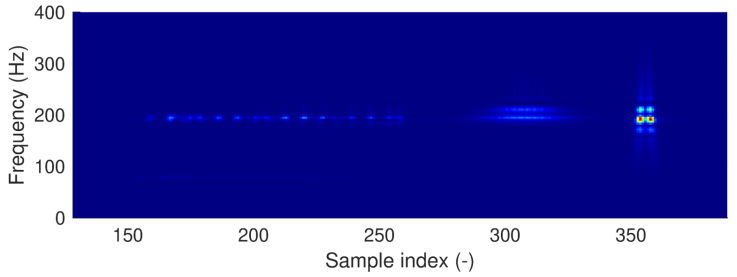

(a) Short-time DZT zoom spectrum modul.

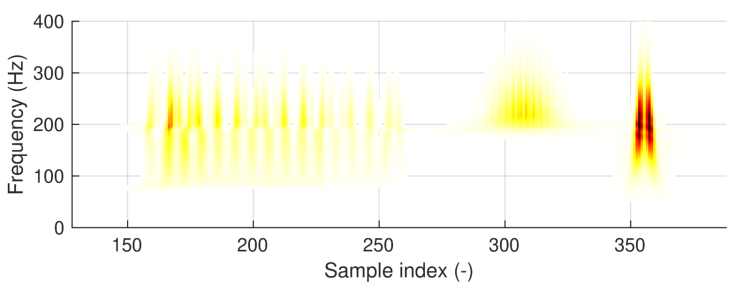

(b) Local selectivity index $K$ modul.

Fig. 7. Short-time DZT zoom spectrum of the signal Fig. 4; the center frequency at $\approx 190 \mathrm{~Hz} \rightarrow k^{\prime}=0.04$; Hamming window is used prior to the spectrum computation; window length of 256 samples and window step of 1 sample.

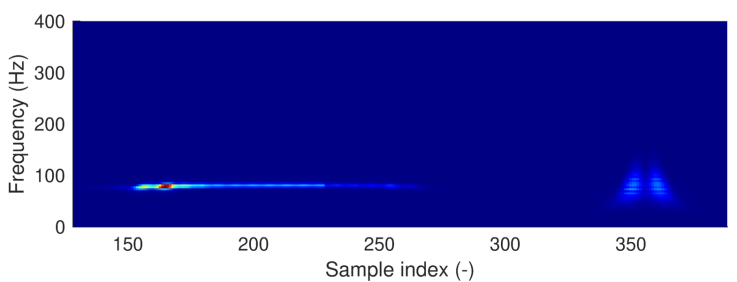

(a) Short-time DZT zoom spectrum modul.

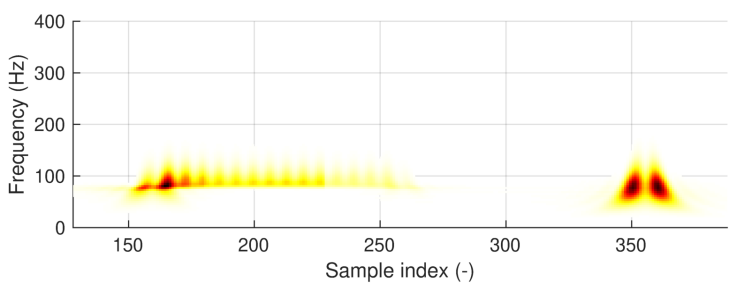

(b) Local selectivity index $K$ modul.

Fig. 8. Short-time DZT zoom spectrum of the signal Fig. 4; the center frequency at $\approx 80 \mathrm{~Hz} \rightarrow k^{\prime}=0.07$; Hamming window is used prior to the spectrum computation; window length of 256 samples and window step of 1 sample.

\section{REFERENCES}

[1] V. I. Lebedev, "Zolotarev polynomials and extremum problems," Russian Journal of Numerical Analysis and Mathematical Modelling, vol. 9, no. 3, pp. 231-264, 1994.

[2] C. Detaille and J. P. Thiran, "Complex Zolotarev polynomials on the real interval $[-1,1]$," Journal of Approximation Theory, vol. 72, no. 3, pp. 317-328, 1993.

[3] G. Grasegger and N. T. Vo, "An algebraic-geometric method for computing Zolotarev polynomials," in Proceedings of the 2017 ACM on International Symposium on Symbolic and Algebraic Computation, ser. ISSAC '17. New York, NY, USA: Association for Computing Machinery, 2017, p. 173-180.

[4] F. Peherstorfer, "Asymptotic representation of Zolotarev polynomials," Journal of the London Mathematical Society, vol. 74, no. 1, pp. 143-153, 2006.

[5] R. P. Boas, "Extremal problems for polynomials," The American Mathematical Monthly, vol. 85, no. 6, pp. 473-475, 1978.

[6] C. Xiangkun and T. Parks, "Analytic design of optimal FIR narrow-band filters using Zolotarev polynomials," IEEE Transactions on Circuits and Systems, vol. 33, no. 11, pp. 10651071, 1986.

[7] M. Vlček and R. Unbehauen, "Zolotarev polynomials and optimal FIR filters," IEEE Transactions on Signal Processing, vol. 47, no. 3, pp. 717-730, Mar 1999.
[8] A. Mingazin, S. Gordienko, and A. Gureev, "IIR filter design: tolerance initial parameter space of Zolotarev-Cauer filters," Components \& Technologies, pp. 122-126, 102016.

[9] M. Vlček, J. Janík, V. Turoň, P. Sovka, and Špetík R., "A way to a new multi-spectral transform," in World Scientific and Engineering Academy and Society (WSEAS)' 11 and GAVTASC'11, Stevens Point, Wisconsin, USA, 2011, pp. 177-182.

[10] M. Vlček, P. Sovka, and R. Špetík, "Algebraic Solutions for Iso-extremal Polynomials on Two Disjoint Intervals," in 9th IMA International Conference on Mathematics in Signal Processing, The Institute of Mathematics and its Applications. 16 Nelson Street, Southend on Sea, Essex SS1 1EF: Catherine Richards House, 122012.

[11] V. Turoň, J. Janík, Špetík R., P. Sovka, and M. Vlček, "Study of ADZT properties for spectral analysis," in World Scientific and Engineering Academy and Society (WSEAS)' 11 and GAVTASC'11, Stevens Point, Wisconsin, USA, 2011, pp. 171-176.

[12] V. Turoň, "Description of spectral analysis based on Zolotarev polynomials (in Czech)," Ph.D. dissertation, Czech Technical University in Prague, Faculty of Electrical Engineering, 82016.

[13] B. Bajpeyee and S. Sharma, "Detection of bearing faults in induction motors using short time approximate discrete Zolotarev transform," in International Conference on Signal Processing (ICSP 2016), 11 2016, pp. 1-7.

[14] R. Špetík, "The discrete Zolotarev transform," Ph.D. dissertation, Czech Technical University in Prague, Faculty of Electrical Engineering, 22009.

[15] J. Kubák, P. Sovka, and M. Vlček, "Evaluation of computing symmetrical zolotarev polynomials of the first kind," Radioengineering, vol. 26, pp. 903-913, 092017.

[16] J.-S. Lee, T.-M. Yoon, and K.-B. Lee, "Bearing fault detection of IPMSMs using zoom FFT," Journal of Electrical Engineering and Technology, vol. 11, no. 5, p. 1235-1241, 92016.

[17] M. Ortigueira, A. Serralheiro, and J. Tenreiro Machado, "A new zoom algorithm and its use in frequency estimation," Waves, Wavelets and Fractals, vol. 1, 012015. 